\title{
Enhanced encapsulation of metoprolol tartrate with carbon nanotubes as adsorbent
}

\author{
Kevin Garala $\cdot$ Jaydeep Patel $\cdot$ Anjali Patel $\cdot$ \\ Abhay Dharamsi
}

Received: 7 June 2011/Accepted: 7 September 2011 / Published online: 27 September 2011

(c) The Author(s) 2011. This article is published with open access at Springerlink.com

\begin{abstract}
A highly water-soluble antihypertensive drug, metoprolol tartrate (MT), was selected as a model drug for preparation of multi-walled carbon nanotubes (MWCNTs)impregnated ethyl cellulose (EC) microspheres. The present investigation was aimed to increase encapsulation efficiency of MT with excellent adsorbent properties of MWCNTs. The unique surface area, stiffness, strength and resilience of MWCNTs have drawn much anticipation as carrier for highly water-soluble drugs. Carbon nanotubes drug adsorbate (MWCNTs:MT)-loaded EC microspheres were further optimized by the central composite design of the experiment. The effects of independent variables (MWCNTs:MT and EC:adsorbate) were evaluated on responses like entrapment efficiency (EE) and $t_{50}$ (time required for $50 \%$ drug release). The optimized batch was compared with drug alone EC microspheres. The results revealed high degree of improvement in encapsulation efficiency for MWCNTs:MT-loaded EC microspheres. In vitro drug release study exhibited complete release form drug alone microspheres within $15 \mathrm{~h}$, while by the same time only $50-60 \%$ drug was released for MWCNTsimpregnated EC microspheres. The optimized batch was further characterized by various instrumental analyses such as scanning electron microscopy, powder X-ray diffraction and differential scanning calorimetry. The results endorse encapsulation of MWCNTs:MT adsorbate inside the matrix of EC microspheres, which might have resulted in
\end{abstract}

K. Garala $(\bowtie) \cdot$ J. Patel · A. Dharamsi

Department of Pharmaceutics, Atmiya Institute of Pharmacy,

Kalawad Road, Rajkot 360005, Gujarat, India

e-mail: kevincgarala@gmail.com

A. Patel

Department of Pharmaceutics, R. D. Gardi B. Pharmacy College, Nyara, Rajkot, Gujarat, India enhanced encapsulation and sustained effect of MT. Hence, MWCNTs can be utilized as novel carriers for extended drug release and enhanced encapsulation of highly watersoluble drug, MT.

Keywords Multi-walled carbon nanotubes . Central composite design · Ethyl cellulose . In vitro drug release $\cdot$ Microspheres

\section{Introduction}

High water solubility of the active pharmaceuticals necessitates a controlled and sustained release medication (Choudhury and Kar 2009). There are several drug delivery systems available to sustain the release rate of highly watersoluble drugs. Among them, microencapsulation technique has been a prime choice by most researchers (Yang et al. 2001; Luzzi and Palmier 1984). Microencapsulation being a multi-unit particulate system bypasses the variations associated with gastric emptying and different transit time (Ghebre-Sellassie 1994). The uniform distribution of multiparticulate system along the gastrointestinal tract (GIT) could lead to more reproducible drug absorption and minimize risk of local irritation as compared to single unit dosage form (Galeone et al. 1981). Numerous methods have been used to prepare polymeric microparticulate drug delivery systems of highly water-soluble drugs (Benita et al. 1984; Bodmeier and McGinity 1987a, b; Jeffery et al. 1993; Amperiadou and Georgarakis 1995a, b; Genta et al. 1997; Schlicher et al. 1997; Ghorab et al. 1990; Cheu et al. 2001). Different polymeric materials have been enveloped to formulate microparticulate systems (microspheres), which include chitosan, gelation, alginate, ethyl cellulose, D-L-lactide-co-glycolide and their copolymers (Jeyanthi and 
Panduranga Rao 1988; Hazrati and DeLuca 1989) Ethyl cellulose being a biocompatible and water-insoluble polymer is one of the extensively studied encapsulating materials for sustained release (Benita and Donbrow 1982; Amperiadou and Georgarakis 1995a, b; Yang et al. 2000; Wade and Weller 1994; Chowdary et al. 2004). This is attributed to its high safety, stability, easy fabrication and low cost. Acetone was selected as a solvent for emulsification processes based on human safety concern over the disadvantageous impact of residue solvent (Sah 1997; Allemann et al. 1993). Unfortunately, the number of shortcomings associated with this carrier includes high drug leakage, low mechanical strength and serious swelling due to their open structure and large pore size (Choi and Han 2005; Ito et al. 2008; O'Donnell and McGinity 1997; Blandino et al. 2000; Blandino et al. 2001; Bajpai and Saxena 2004; Boonsongrit et al. 2008). These drawbacks restrict the use of EC as a polymer for microencapsulation. Various attempts have been done to overcome the problems associated with EC, which includes cross linking (Anal and Stevens 2006), solvent evaporation (Yang et al. 2005), ion exchange (Abdekhodaie and $\mathrm{Wu} 2008$ ) and surface coating (Zhang et al. 2008), but to date none of them have shown distinguished effectiveness.

Nanotechnology offers lots of openings and benefits for new drug molecules with extensively enhanced characteristics. Pharmaceutical innovations have chiefly concentrated on the design of modified release drug delivery systems using diverse newly discovered excipients. In the last few years, several carriers have been developed for effective drug delivery of highly water-soluble drugs. Carbon nanotubes (CNTs), being one of such carriers, represent allotropes of carbon with cylindrical tubulesshaped concentric graphitic (a hexagonal lattice of carbon) sheets and capped by fullerene-like hemispheres with a length to diameter ratio $>1,000,000$ (Iijima 1991). Their inert nature (Bonard et al. 1998; Niyogi et al. 2002), large surface area (Wu and Liao 2007), strong hydrophobic property (Tagmatarchis and Prato 2005) and available flexibility for physiochemical manipulation (Tasis et al. 2006) have sparked much interest in the pharmaceutical scientist. Wide amount of research is dedicated to their innovative applications in the design of the modified release dosage form. CNTs are mechanically stable substances with special ability to incorporate material into their inner hollow spaces (Ajima et al. 2005) or adsorption over the surface (Davydov et al. 2005). CNTs-doped microspheres of nicotinamide adenine dinucleotide with increased loading efficiency were developed by $\mathrm{Lu}$ et al. (2005). Jiang et al. (2006) have also developed microspheres containing bovine serum albumin (BSA) using CNTs as a carrier to overcome the drawbacks of polymeric microspheres. They observed considerable reduction of
BSA leakage with dramatic increase in the strength of microspheres. In light of these, CNTs-impregnated microspheres may effectively overcome the drawbacks associated with microspheres containing water-soluble drug.

Metoprolol tartrate (MT) is a highly water-soluble antihypertensive drug with a half-life of 3-4 h. The short biological half-life provides suitability of the drug for sustained drug delivery. The basic nature attributes to high $\mathrm{pKa}$ value (9.5) of the drug. It is prescribed alone or in combination with other antihypertensive agents for the long-term management of angina pectoris and myocardial infarction. Moreover, it is readily and completely absorbed from the GIT with a bioavailability of about 50\% (Martindale 2009; Chrysant 1998). Since MT requires multiple daily dosage to maintain adequate plasma concentrations, it was selected as a model drug for sustained release polymeric microparticulate formulation using MWCNTs as adsorbent (Dadashzadeh et al. 2003; Gambhire et al. 2007).

Hence, the present investigation aimed at preparing MWCNTs-impregnated EC microspheres of a highly water-soluble drug, MT, to achieve sustained release with elimination of multiple daily dosing.

\section{Materials and methods}

\section{Materials}

MT was obtained as a gift sample from Sun Pharmaceutical (Mumbai, India). Multi-walled carbon nanotubes (MWCNTs) were procured from Nanoshel LLI (Wilmington, USA). Ethyl cellulose (EC) and Tween 80 were obtained from Hi-Media (Mumbai, India). Other chemicals were of analytical grade and used as received without further modification. Deionized water was used throughout the study.

\section{Adsorption isotherm}

Several reports demonstrated that CNTs had good adsorption capacities for different materials due to their hollow and layered nanosized structures that have a large specific surface area (Long and Yang 2001; Li et al. 2002; Peng et al. 2003). MT solutions were prepared by dissolving solid in deionized water. Batch adsorption studies were performed in glass bottles with MT solution $(100 \mathrm{~mL})$ of the prescribed concentration, ranging from 5 to $50 \mathrm{mg} \mathrm{dm}^{-3}$ (5 $\mathrm{mg} \mathrm{dm}^{-3}$ interval), and adsorbent material (MWCNTs, $0.1 \mathrm{~g}$ ) was added to each bottle. The bottles were capped with glass stoppers followed by sonication for $5 \mathrm{~min}$ and subsequently subjected to isothermal shaking incubator (Nova, Mumbai, India) for $24 \mathrm{~h}$ at $30^{\circ} \mathrm{C}$. After equilibration, the suspension was filtered through a 
$0.22-\mu \mathrm{m}$ filter and the filtrate was analyzed for the presence of any unadsorbed MT content by double beam UV/visible spectrophotometer (Shimadzu 1700, Tokyo, Japan) at $276 \mathrm{~nm}$ (Shinde et al. 2009). The amount of MT absorbed by the adsorbent material (MWCNTs) was calculated based on a mass balance equation as given below:

$q=\frac{\left(C_{0}-C_{\mathrm{eq}}\right) \times V}{W}$

where $q$ is the amount of MT adsorbed by MWCNTs, $\mathrm{mg} \mathrm{g}^{-1} ; C_{0}$ and $C_{\text {eq }}$ are the initial and equilibrium concentration of MT in the solution, $\mathrm{mg} \mathrm{dm}^{-3} ; V$ is the solution volume, $\mathrm{dm}^{3}$; and $W$ is the dry weight of adsorbent, $\mathrm{g}$.

\section{Adsorption isotherm models}

Literature survey has cited various examples of drug adsorption on the various carriers. So far, kaolin (Afolabi 2006), chitosan (Alkhamis 2001) and antacids (Aideloje et al. 1998) have been studied for drug adsorption, and it has been observed that depending on the surface area and nature of the adsorbent, the extent of adsorption varies. Langmuir, Freundlich and Sips adsorption isotherm has been used to study the same. In the case of adsorption of drug on MWCNTs, the same method has been employed to study drug adsorption by MWCNTs.

The representation of the Langmuir model is given as:

$q_{\mathrm{e}}=\frac{q_{\mathrm{m}} K_{\mathrm{L}} C_{\mathrm{e}}}{1+K_{\mathrm{L}} C_{\mathrm{e}}}$

where $q_{\mathrm{e}}$ is the equilibrium MT concentration on the adsorbent, $\mathrm{mg} \mathrm{g}^{-1} ; C_{\mathrm{e}}$ is the equilibrium concentration of MT in the solution, $\mathrm{mg} \mathrm{l}^{-1} ; q_{\mathrm{m}}$ is related to the maximum adsorption capacity of adsorbent $\mathrm{mg} \mathrm{g}^{-1}$; and $K_{\mathrm{L}}$ is the constant term related to energy of adsorption, $\mathrm{dm}^{3} \mathrm{mg}^{-1}$.

The representation of the Freundlich model is:

$q_{\mathrm{e}}=K_{\mathrm{F}} C_{\mathrm{e}}^{1 / n}$

where $K_{\mathrm{F}}$ is the Freundlich constant related to the adsorbent capacity, $\mathrm{dm}^{3} \mathrm{~g}^{-1}$, and $1 / n$ measures the surface heterogeneity.

Sips model is a combination of Langmuir and Freundlich models and is stated as:

$q_{\mathrm{e}}=\frac{q_{\mathrm{max}} K_{\mathrm{eq}} C_{\mathrm{e}}^{n}}{1+K_{\mathrm{eq}} C_{\mathrm{e}}^{n}}$

where $K_{\text {eq }}$ represents the equilibrium constant of Sips equation, $\mathrm{dm}^{3} \mathrm{mg}^{-1}$; and $q_{\max }$ is the maximum adsorption capacity, $\mathrm{mg} \mathrm{g}^{-1}$. Sips isotherm model is distinguished by the heterogeneity factor, $n$. Data obtained from adsorption isotherm method were fitted to the Langmuir, Freundlich and Sips isotherm models using a method of least square. From the nonlinear regression coefficient $\left(R^{2}\right)$ and nonlinear Chi-square test $\left(\chi^{2}\right)$ value, the best fit model was predicted.

Preparation of drug-nanotubes adsorbate

Drug-nanotubes adsorbate (MT:MWCNTs) was prepared by the nanoprecipitation method (Ajima et al. 2004). Accurately weighed amount of MT was dissolved in $25 \mathrm{~mL}$ of deionized water followed by addition of MWCNTs in the required quantity. The mixture was further subjected to sonication (Frontline FS-4, Mumbai, India) for $15 \mathrm{~min}$ followed by incubation in hot air oven (Nova, Mumbai, India) at $40^{\circ} \mathrm{C}$ for $48 \mathrm{~h}$. The residue left after evaporation (MWCNTs:MT) was analyzed for drug content by the UV method at $276 \mathrm{~nm}$.

\section{Drug content estimation of adsorbate}

As much as $10 \mathrm{mg}$ of adsorbate was added to $50 \mathrm{~mL}$ of deionized water with subsequent sonication for $30 \mathrm{~min}$. The resultant solution was kept at ambient temperature for $24 \mathrm{~h}$ for complete extraction of MT from the adsorbate. The dispersion was filtered through Whatman filter paper (Sartorius Stedim, Bangalore, India) and analyzed by UV spectroscopy.

Preparation of microspheres

MWCNTs-impregnated EC microspheres of MT were prepared by the solvent evaporation technique (Cheu et al. 2001). Briefly, ethyl cellulose was dissolved in $25 \mathrm{~mL}$ of acetone along with the prepared adsorbate. The dispersion was further subjected to sonication for 15 min with intermediate shaking. The resultant dispersion was added dropwise to $250 \mathrm{~mL}$ of light liquid paraffin (containing $0.2 \% \mathrm{w} / \mathrm{v}$ Tween 80 ) with continuous stirring by magnetic stirrer (Remi Labs, Mumbai, India). After $2 \mathrm{~h}$, microspheres were collected and washed with petroleum ether with subsequent air drying at room temperature. A similar method was utilized for preparing EC microspheres of MT without incorporation of MWCNTs.

Experimental design

The MWCNTs:MT ratio and polymer concentration play a crucial role in the preparation of MWCNTs-impregnated EC microspheres of MT. Center composite design (CCD) was employed for systemic study of joint influence of the effect of independent variables [MWCNTs:MT $\left(X_{1}\right)$ and EC:drug adsorbate $\left.\left(X_{2}\right)\right]$ on responses such as drug entrapment efficiency (EE) and time for 50\% drug release $\left(t_{50}\right)$. The selections of dependent variables were done on the basis of the aim of the present investigation (enhanced 
encapsulation and sustained drug release). Based on preliminary trials, two factors were determined as follows: MWCNTs:MT ratio $\left(X_{1}\right)$ : $0.25-2.75$ and EC:drug adsorbate ratio $\left(X_{2}\right): 0.10-0.30$. In this design, two factors with five levels were probed to investigate the main effects and interaction of the two factors on two responses (Table 1). The design consists of nine runs (4 factorial points, 4 star points and 1 center point) and four replicated runs (center points) yielding 13 experiments in total. The main purpose of the replication runs was to increase the precision and to minimize experimental error. A third-order quadratic model incorporating interactive and polynomial terms was used to evaluate the response.

$$
\begin{aligned}
Y_{i}= & b_{0}+b_{1} X_{1}+b_{2} X_{2}+b_{3} X_{1}^{2}+b_{4} X_{2}^{2}+b_{5} X_{1} X_{2} \\
& +b_{6} X_{1}^{2} X_{2}+b_{7} X_{1} X_{2}^{2}
\end{aligned}
$$

where, $Y_{i}$ was the dependent variable, $b_{0}$ was arithmetic mean response of the 13 runs and $b_{i}$ was the estimated coefficient for factor $X_{i}$. The main effects $\left(X_{1}\right.$ and $\left.X_{2}\right)$ represent the average result of changing one factor at a time from its low to high value. The interaction terms $\left(X_{1} X_{2}\right)$ show how the response changes when two factors are simultaneously changed. The polynomial terms $\left(X_{1}^{2}\right.$ and $X_{2}^{2}$ ) were included to investigate nonlinearity (Liu et al. 2009).

Data were further analyzed by Microsoft Excel ${ }^{\circledR}$ for regression analysis. Analysis of variance (ANOVA) was implemented to assure that there was no significant difference between the developed full model and the reduced model. Response surface plots were plotted to study response variations against two independent variables using Design Expert $^{\circledR}$ Version 8 software.

Table 1 Central composite design batches

\begin{tabular}{lll}
\hline Batch & $\begin{array}{l}\text { MWCNTs:MT } \\
\left(X_{1}\right)\end{array}$ & $\begin{array}{l}\text { EC:adsorbate } \\
\left(X_{2}\right)\end{array}$ \\
\hline 1 & 1 & -1 \\
2 & -1 & 1 \\
3 & $-\alpha^{\mathrm{a}}$ & 0 \\
4 & -1 & -1 \\
5 & 0 & $-\alpha$ \\
6 & $\alpha$ & 0 \\
7 & 1 & 1 \\
8 & 0 & $\alpha$ \\
$9-13$ & 0 & 0 \\
\hline
\end{tabular}

\begin{tabular}{llllll}
\hline Factor & \multicolumn{2}{l}{ Level } & & & \\
\cline { 2 - 6 } & $-\alpha$ & -1 & 0 & 1 & $\alpha$ \\
\hline$X_{1}$ (MWCNTs:MT) & 0.25 & 0.62 & 1.50 & 2.38 & 2.75 \\
$X_{2}$ (EC:adsorbate) & 0.10 & 0.13 & 0.20 & 0.27 & 0.30
\end{tabular}

${ }^{\mathrm{a}} \alpha=1.414$
Characterization of microspheres

\section{Yield of microspheres}

Fresh microspheres impregnated with MWCNTs and of drug alone were collected and weighed separately. The measured weight of microspheres was divided by the total amount of all nonvolatile components that were used for the preparation (Patel et al. 2006).

$\%$ Yield $=\frac{\text { Actual weight of product }}{\text { Total weight of excipients and drug }} \times 100$

Entrapment efficiency (EE)

Intact microspheres (equivalent to $100 \mathrm{mg}$ of drug) were crushed and extracted with methanol. The extract was further filtered by Whatman filter paper (Sartorius Stedim, Bangalore, India) and diluted to $100 \mathrm{~mL}$ with methanol. The drug content of filtrate was determined spectrophotometrically at $276 \mathrm{~nm}$ (Patel et al. 2006). The amount of drug entrapped in the microspheres was calculated as:

$\mathrm{EE}=\frac{\text { Practical drug content }}{\text { Theoretical drug load expected }} \times 100$

\section{Micromeritics}

Sphericity of microspheres Photomicrographs of randomly selected microspheres were utilized to calculate the area $(A)$ and perimeter $(P)$ of the microspheres. The shape of the microspheres was estimated by computing the shape factor and circularity factor (Jadhav et al. 2007; Singh et al. 2007). It was calculated by the following equation.

Shape factor $=P^{\prime} / P, \quad$ where $P^{\prime}=2 \pi(A / \pi)^{1 / 2}$

Circularity factor $=P^{2} / 12.56 \times A$

Determination of flow properties The flow behavior of microspheres was quantified by the angle of repose and Carr's Index (Lee et al. 2000; Parul et al. 2008).

\section{Instrumental analysis}

Scanning electron microscopy (SEM) Surface topography of MWCNTs, drug adsorbate and optimized batch of microspheres were observed under a scanning electron microscope (Model JSM 5610LV, Jeol, Japan). The samples were attached to the slab surfaces with double-sided adhesive tapes and the scanning electron photomicrograph was taken at $1,000-15,000 \times$ magnification.

Powder X-ray diffraction (PXRD) Samples of MT, MWCNTs, adsorbate, EC microspheres (without MWCNTs) and EC microspheres impregnated with MWCNTs were subjected to X-ray diffraction (PANalytical X'pert PRO- 
6340, India) to investigate their X-ray diffraction patterns. The data were recorded over a range of $2^{\circ}-100^{\circ}$ at a scanning rate of $5 \times 10^{3} \mathrm{cps}$ using a chart speed of $5 \mathrm{~mm}$ per $2^{\circ}$.

Differential scanning calorimetry (DSC) $5-10 \mathrm{mg}$ of fresh samples as mentioned for PXRD were studied by differential scanning calorimeter (Linseis STA PT-1600, Germany). The samples were hermetically sealed in an aluminum crucible before analysis. The system was purged with nitrogen gas at a flow rate of $60 \mathrm{~mL} \mathrm{~min}{ }^{-1}$. Heating was done between 30 and $300^{\circ} \mathrm{C}$ at a rate of $10^{\circ} \mathrm{C} \mathrm{min}{ }^{-1}$.

\section{In vitro drug release}

Accurately weighed amount of microspheres (equivalent to $100 \mathrm{mg}$ of drug) from each batch were subjected to dissolution studies using USP dissolution test apparatus type I in $900 \mathrm{~mL}$ of $\mathrm{pH} 6.8$ phosphate buffer maintained at $37 \pm 0.5^{\circ} \mathrm{C} ; 5 \mathrm{~mL}$ aliquots were withdrawn at 10 -min time intervals for the first hour while the remaining samples were withdrawn at 60-min time intervals for $24 \mathrm{~h}$. Each sampling was followed by the simultaneous addition of fresh medium. The samples were filtered through Whatman filter paper (Sartorius Stedim, Bangalore, India) before being analyzed for the amount of drug released.

\section{Results and discussion}

\section{Adsorption isotherm of MWCNTs for MT}

Adsorption isotherm of MWCNTs for MT was studied using Langmuir, Freundlich and Sips adsorption isotherm models. The results of nonlinear $R^{2}$ and $\chi^{2}$ for the three adsorption isotherms are shown in Table 2. The maximum adsorption capacity $\left(q_{\mathrm{m}}\right)$ of the MWCNTs for MT was $443.04 \mathrm{mg} \mathrm{g}^{-1}$ (Table 2). The $R_{\mathrm{L}}$ value of the MWCNTs (0.028) obtained from the Langmuir isotherm model for the initial MT concentration of $50 \mathrm{mg} \mathrm{dm}^{-3}$ indicated favorable adsorption of MT onto MWCNTs. The adsorption isotherm data for MT exhibited good fit with Langmuir model $\left(R^{2}=0.9971\right)$ and Sips model $\left(R^{2}=0.9969\right)$ than the Freundlich model $\left(R^{2}=0.8542\right)$. The results of the nonlinear $\chi^{2}$ for the three adsorption isotherms indicated that the Langmuir isotherm model appeared to be the best fitting model for the adsorption isotherm data of the MWCNTs, because it displayed the lowest Chi-square, $\chi^{2}$ (5.43) with the highest $R^{2}(0.9971)$ values. The value of $n$ for MT adsorption onto MWCNTs was close to unity ( $n=1.005)$, indicating homogeneous adsorption. Also, there was close similarity between the maximum adsorption capacity values obtained from Langmuir (443.04 $\mathrm{mg} \mathrm{g}^{-1}$ ) and Sips (444.67 $\mathrm{mg} \mathrm{g}^{-1}$ ) isotherm models (Table 2). From the adsorption isotherm data, it is concluded that the
Table 2 Constant for equilibrium isotherm models with error analysis values for MT:MWCNTs adsorbate

Isotherm model Parameter value (at constant temperature, $30^{\circ} \mathrm{C}$ )

\begin{tabular}{llllll}
\cline { 2 - 5 } & $K_{\mathrm{L}}$ & $q_{\mathrm{m}}$ & $R_{\mathrm{L}}$ & $R^{2}$ & $\chi^{2}$ \\
\hline Langmuir & 0.0572 & 443.04 & 0.028 & 0.9971 & 5.43 \\
\hline
\end{tabular}

Isotherm model Parameter value (at constant temperature, $30^{\circ} \mathrm{C}$ )

\begin{tabular}{lllll} 
& $K_{\mathrm{F}}$ & $1 / n$ & $R^{2}$ & $\chi^{2}$ \\
\hline Freundlich & 15.7575 & 0.3547 & 0.8542 & 78.45
\end{tabular}

Isotherm model Parameter value (at constant temperature, $30^{\circ} \mathrm{C}$ )

\begin{tabular}{llllll} 
& $q_{\max }$ & $K_{\text {eq }}$ & $n$ & $R^{2}$ & $\chi^{2}$ \\
\hline Sips & 444.67 & 0.042 & 1.005 & 0.9969 & 14.31 \\
\hline
\end{tabular}

$K_{L}$ constant term related to energy of adsorption, $\mathrm{dm}^{3} \mathrm{mg}^{-1} ; q_{m}$ maximum adsorption capacity of adsorbent, $\mathrm{mg} \mathrm{g}^{-1} ; R_{L}$ adsorption coefficient; $R^{2}$ regression coefficient; $\chi^{2}$ Chi-square value; $K_{F}$ Freundlich constant, $\mathrm{dm}^{3} \mathrm{~g}^{-1} ; q_{\max }$ maximum adsorption capacity, $\mathrm{mg} \mathrm{g}^{-1} ; K_{e q}$ equilibrium constant, $\mathrm{dm}^{3} \mathrm{mg}^{-1} ; n$ heterogeneity factor

MWCNTs is a good adsorbent for MT with homogeneous adsorption to reduce batch to batch variability.

Experimental design (CCD)

Preliminary investigations of the process parameters revealed that factors such as ratio of MWCNTs:drug $\left(X_{1}\right)$ and EC:drug adsorbate $\left(X_{2}\right)$ exhibited significant influence on rate of entrapment efficiency (EE) and in vitro drug release; hence, they were utilized for further systematic studies. Both selected dependent variables (EE and $t_{50}$ ) for all 13 batches showed a wide variation of $84.17-97.78 \%$ and 5.88-15.05 h, respectively (Table 3). The data clearly

Table 3 Results of experimental design batches

\begin{tabular}{lllll}
\hline Batch & $X_{1}$ & $X_{2}$ & EE $(\%)$ & $t_{50}(\mathrm{~h})$ \\
\hline 1 & 2.38 & 0.13 & $87.42 \pm 0.26$ & $10.89 \pm 0.97$ \\
2 & 0.62 & 0.27 & $92.94 \pm 0.47$ & $09.71 \pm 0.52$ \\
3 & 0.25 & 0.20 & $86.08 \pm 0.18$ & $08.93 \pm 0.65$ \\
4 & 0.62 & 0.13 & $88.04 \pm 0.33$ & $08.42 \pm 0.48$ \\
5 & 1.50 & 0.10 & $84.17 \pm 0.46$ & $05.88 \pm 0.42$ \\
6 & 2.75 & 0.20 & $96.01 \pm 0.81$ & $11.95 \pm 0.78$ \\
7 & 2.38 & 0.27 & $93.84 \pm 0.57$ & $12.83 \pm 0.11$ \\
8 & 1.50 & 0.30 & $97.78 \pm 0.34$ & $15.05 \pm 0.61$ \\
9 & 1.50 & 0.20 & $92.04 \pm 0.42$ & $09.63 \pm 0.45$ \\
10 & 1.50 & 0.20 & $89.97 \pm 0.84$ & $09.61 \pm 0.81$ \\
11 & 1.50 & 0.20 & $90.52 \pm 0.25$ & $09.74 \pm 0.47$ \\
12 & 1.50 & 0.20 & $91.85 \pm 0.55$ & $09.72 \pm 0.48$ \\
13 & 1.50 & 0.20 & $89.47 \pm 1.01$ & $09.91 \pm 0.77$ \\
\hline$X_{y}$ & MN & &
\end{tabular}

$X_{1}$ MWCNTs:MT, $X_{2}$ EC:adsorbate, EE entrapment efficiency, $t_{50}$ time required for $50 \%$ drug release 
indicate the strong influence of $X_{1}$ and $X_{2}$ on selected responses (EE and $t_{50}$ ). The polynomial equations can be used to draw conclusions after considering the magnitude of coefficients and the mathematical sign carried: positive or negative. For EE, coefficients $b_{3}, b_{4}$ and $b_{5}$ were found to be insignificant, as $p$ values were more than 0.05 and hence removed from the full model. Similarly, for $t_{50}$, values of $b_{5}$ and $b_{7}$ were insignificant and hence removed from the full model (Table 4). Table 5 shows the results of analysis of variance (ANOVA) performed to justify the removal of insignificant factors. The high values of correlation coefficients for EE and $t_{50}$ indicate a good fit. The critical values of $\mathrm{F}$ for $\mathrm{EE}$ and $t_{50}$ were found to be 5.41 $(d f=3,5)$ and $5.79(d f=2,5)$, respectively, at $\alpha=0.05$. Moreover, calculated $F$ value [0.1742 (EE), $\left.0.8475\left(t_{50}\right)\right]$ was found to be less than critical value, which suggests no significant difference between the full and reduced model. The data of all the 13 batches of factorial design were used to generate interpolated values using Design Expert ${ }^{\circledR}$ Version 8 software. High levels of both $X_{1}$ and $X_{2}$ were found to be favorable for sustained release and high entrapment efficiency. Multiple linear regression analysis (Table 4) also revealed positive values of coefficient $b_{1}$ and $b_{2}$ for both responses. This indicated that as MWCNTs:drug ratio $\left(X_{1}\right)$ and EC:drug adsorbate ratio $\left(X_{2}\right)$ were increased, there was a significant improvement in EE and $t_{50}$.

\section{Influence of formulation composition factor on entrapment efficiency (EE)}

The major aim of the present investigation was to increase the entrapment efficiency of a highly water-soluble drug, MT. Response surface plot for EE (Fig. 1) illustrated strong influence of two factors (MWCNTs:MT ratio and EC:adsorbate ratio). An EE of $97.78 \%$ was observed with MWCNTs:drug ratio 1.5 and EC:adsorbate ratio 0.30 (Batch 8). This might be attributed to the ability of MWCNTs to prevent leaching of drug after encapsulation inside the matrix of $\mathrm{EC}$ microspheres and also due to increase in the amount of EC in the formulation that ultimately increase the polymeric matrix from which MT has to release.

\section{Influence of formulation composition factor} on in vitro drug release $\left(t_{50}\right)$

A strong influence of both independence variables (MWCNTs: drug ratio and EC: adsorbate ratio) was observed on in vitro drug release $\left(t_{50}\right)$ (Fig. 2). The highest $t_{50}$ value $(15.05 \pm 0.6114)$ was observed with Batch 8 (MWCNTs:MT ratio 1.5 and EC:adsorbate ratio 0.30). It indicates retardation of drug release, which again endorsed the resilience and stiffness of MWCNTs along with increased amount of EC with respect to drug.

Table 5 Calculation of testing the model in portions

\begin{tabular}{cllll}
\hline & DF & SS & MS & $R^{2}$ \\
\hline EE & & & & \\
Regression & & & & \\
FM & 7 & 174.5569 & 24.9367 & 0.9690 \\
RM & 4 & 173.9737 & 43.4934 & 0.9658 \\
Error & & & & \\
FM & 5 & 6.4767 & 1.2953 & \\
RM & 8 & 7.2479 & 0.9059 & \\
$t_{50}$ & & & & \\
Regression & & & & \\
FM & 7 & 59.1275 & 8.4467 & 0.9996 \\
RM & 5 & 58.8046 & 11.7609 & 0.9941 \\
Error & & & & \\
FM & 5 & 0.0232 & 0.0046 & \\
RM & 7 & 0.3465 & 0.0494 &
\end{tabular}

$D F$ degree of freedom, $S S$ sum of squares, $M S$ mean of squares, $R$ regression coefficient

Table 4 Summary of regression analysis

\begin{tabular}{lllllllr}
\hline Coefficients & $b_{0}$ & $b_{1}$ & $b_{2}$ & $b_{11}{ }^{\mathrm{a}}$ & $b_{22}{ }^{\mathrm{a}}$ & $b_{12}{ }^{\mathrm{a}}$ & $b_{112}$ \\
\hline EE & & & & & & & $b_{122}{ }^{\mathrm{a}}$ \\
FM & 90.7700 & 3.5113 & 4.8125 & 0.0249 & -0.0100 & 0.3801 & -1.9825 \\
RM & 90.7792 & 3.5113 & 4.8125 & - & - & - & -1.9825 \\
$t_{50}$ & & & & & & -3.4413 \\
FM & 9.5651 & 1.0678 & 3.2425 & 0.3965 & 0.4090 & 0.1625 & -2.4350 \\
RM & 9.5651 & 1.1132 & 3.2425 & 0.3965 & 0.4090 & - & -2.4350 \\
\hline
\end{tabular}

$F M$ full model, $R M$ reduced model, $E E$ entrapment efficiency, $t_{50}$ time required for $50 \%$ drug release

${ }^{a}$ Response is insignificant at $p=0.05$ 


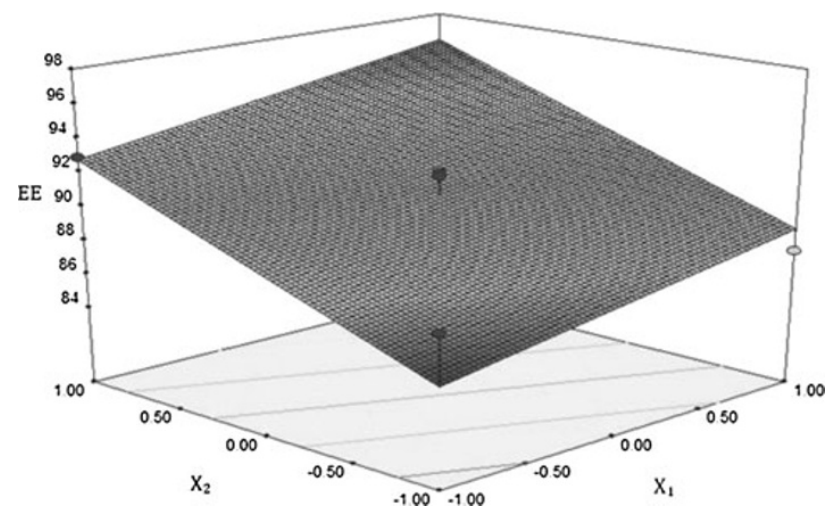

Fig. 1 Response surface plot showing effect of entrapment efficiency of variables [MWCNTs:MT $\left(X_{1}\right)$ and EC:adsorbate $\left(X_{2}\right)$ ]

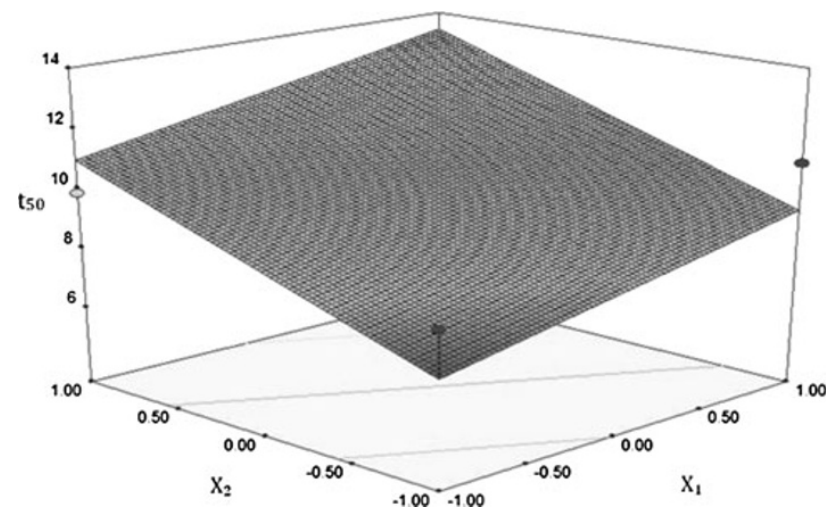

Fig. 2 Response surface plot showing effect of $t_{50}$ (time required for $50 \%$ drug release) of variables [MWCNTs:MT $\left(X_{1}\right)$ and EC:adsorbate $\left.\left(X_{2}\right)\right]$

\section{Characterization}

\section{Yield of microspheres}

The total yield of microspheres was determined by dividing the measured weight with the weight of the total amount of nonvolatile compound. The percentage yield of microspheres of different batches was in the range of 92-97 wt\% (Table 6). Statistically insignificant difference was observed for the total yield of microspheres for microspheres prepared in the presence or absence of MWCNTs, which suggests no major effect of MWCNTs on the yield of microspheres. These results were far better than others previously reported (Shabaraya and Narayanacharyulu 2003).

\section{Entrapment efficiency}

EC microspheres of highly water-soluble drug without incorporation of MWCNTs had very less entrapment efficiency, because most drugs escaped from the polymeric matrix of microspheres (data not shown). The effect of CNTs on the entrapment efficiency of the prepared microspheres was very pronounced (Table 3 ). The entrapment efficiency of the prepared microspheres varied from 84.17 to $97.78 \%$. The entrapment efficiency was increased significantly with increasing MWCNTs concentration, which might be attributed to the interaction between the drug and MWCNTs. It will diminish the overall drug leaching from the formulation with entrapping more amount of drug. The entrapment efficiency was found to be highest for Batch 8 . These results were far better than those previously reported 38.9-72.1\% (Shabaraya and Narayanacharyulu 2003).

Table 6 Characterization of experimental design batches

\begin{tabular}{llllll}
\hline Batch & Yield $(\%)$ & Angle of repose & Carr's Index & Shape factor & Circularity factor \\
\hline 1 & $95.58 \pm 0.41$ & $21.34 \pm 0.17$ & $21.56 \pm 0.65$ & $1.142 \pm 0.048$ & $0.948 \pm 0.004$ \\
2 & $96.08 \pm 0.45$ & $19.45 \pm 0.21$ & $27.64 \pm 0.55$ & $1.265 \pm 0.063$ & $0.952 \pm 0.065$ \\
3 & $94.72 \pm 0.61$ & $18.51 \pm 0.66$ & $29.65 \pm 0.41$ & $1.366 \pm 0.016$ & $0.964 \pm 0.065$ \\
4 & $94.14 \pm 1.04$ & $22.21 \pm 1.21$ & $19.14 \pm 0.58$ & $1.165 \pm 0.016$ & $1.065 \pm 0.015$ \\
5 & $96.45 \pm 1.48$ & $26.14 \pm 0.54$ & $31.15 \pm 1.66$ & $1.054 \pm 0.061$ & $1.056 \pm 0.098$ \\
6 & $93.56 \pm 1.03$ & $25.42 \pm 0.45$ & $28.15 \pm 1.54$ & $0.954 \pm 0.121$ & $1.118 \pm 0.051$ \\
7 & $95.21 \pm 1.15$ & $26.41 \pm 0.14$ & $26.54 \pm 0.19$ & $0.969 \pm 0.015$ & $1.061 \pm 0.05$ \\
8 & $97.22 \pm 0.34$ & $18.15 \pm 0.30$ & $15.04 \pm 0.14$ & $1.012 \pm 0.004$ & $1.001 \pm 0.004$ \\
9 & $96.18 \pm 0.47$ & $29.16 \pm 0.66$ & $32.65 \pm 0.48$ & $1.018 \pm 0.015$ & $0.965 \pm 0.061$ \\
10 & $95.05 \pm 1.42$ & $29.07 \pm 1.31$ & $31.45 \pm 0.21$ & $1.041 \pm 0.016$ & $0.951 \pm 0.065$ \\
11 & $92.77 \pm 0.45$ & $29.97 \pm 1.15$ & $27.00 \pm 0.67$ & $0.984 \pm 0.026$ & $0.916 \pm 0.065$ \\
12 & $94.13 \pm 1.14$ & $31.08 \pm 0.15$ & $29.15 \pm 0.64$ & $0.949 \pm 0.014$ & $1.042 \pm 0.056$ \\
13 & $95.45 \pm 1.11$ & $27.45 \pm 0.16$ & $28.58 \pm 0.34$ & $0.985 \pm 0.055$ & $1.052 \pm 0.067$ \\
\hline
\end{tabular}

Results are mean of triplicate observations $\pm \mathrm{SD}$ 


\section{Micromeritics}

The formed microspheres of all batches were very good in shape and circularity. A statistically insignificant difference was observed for all batches of microspheres, indicating that the shape and circularity of the microspheres (Table 6) were not significantly influenced by MWCNTs. According to the literature, microspheres with $\mathrm{CI}$ values between 5 and $15 \%$ have very good flowability. A statistically insignificant difference was observed in values of Carr's Index for different batches of microspheres (Table 6) suggesting that microspheres of all batches have an excellent flowability irrespective of the presence of MWCNTs. Moreover, the angle of repose of most of the batches indicated a very good flow, which suggested no significant effect of MWCNTs on the flow properties of microspheres. Furthermore, an excellent flow property of microspheres indicates an absence of aggregation and ease of handling. This will help in producing a uniform batch of microspheres for oral delivery.

\section{Instrumental analysis}

Scanning electron microscopy (SEM) The surface morphology of pure MWCNTs, drug adsorbate and MWCNTsimpregnated EC microspheres was determined by scanning electron microscopy (SEM) (Figs. 3, 4, 5). SEM confirmed the fiber-like structure of pure MWCNTs, drug absorbance on the surface of MWCNTs and spherical structure of MWCNTs-impregnated EC microspheres. From the photograph of microspheres, it was quite clear that drug adsorbate was trapped inside the microspheres, which might prevent drug diffusion easily.

Powder X-Ray diffraction X-ray diffraction pattern of MT, MWCNTs, drug adsorbate and EC microspheres with and without MWCNTs impregnation are illustrated in Fig. 6. The X-ray diffractogram of pure MT had sharp peaks at diffraction angles $(2 \theta)$ of around $12^{\circ}, 19^{\circ}, 21^{\circ}$ and

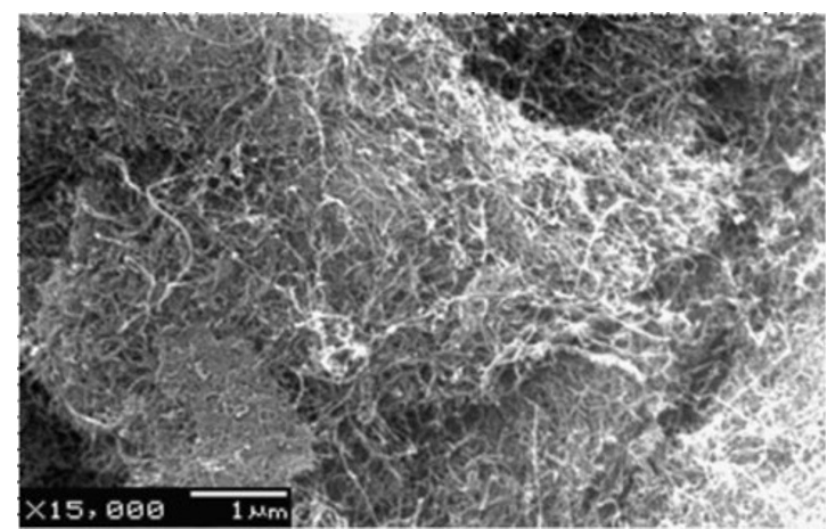

Fig. 3 SEM of MWCNTs $26^{\circ}$, indicating a typical crystalline pattern. The spectrum of MWCNTs showed three reflections of higher intensity at $2 \theta$ of around $26^{\circ}, 43^{\circ}$ and $72^{\circ}$. The X-ray pattern of drug adsorbate indicated characteristics peaks of both MT and MWCNTs with slight poor reflection in the range of $5^{\circ}-75^{\circ}$. All major characteristic crystalline peaks of MT appear in the diffractogram of adsorbates, indicating the absence of any significant conversion of crystalline state. The lower intensity of the drug peaks in the diffractogram of adsorbate was attributed to the physical interaction of the drug and MWCNTs. MWCNTs-impregnated EC microspheres exhibited complete absence of sharp peaks of pure MT, which suggest decreased crystallinity and may be responsible for enhanced encapsulation of drug inside the matrix of EC.

Differential scanning calorimetry (DSC) Differential scanning calorimetry gives reliable information on the physicochemical state of the ingredients of microspheres

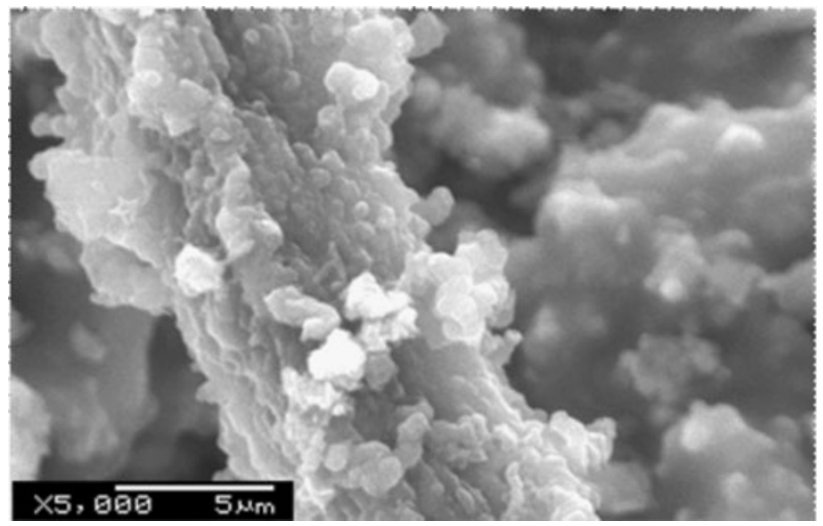

Fig. 4 SEM of MWCNTs:MT adsorbate showing drug (MT) adsorption on surface of MWCNTs

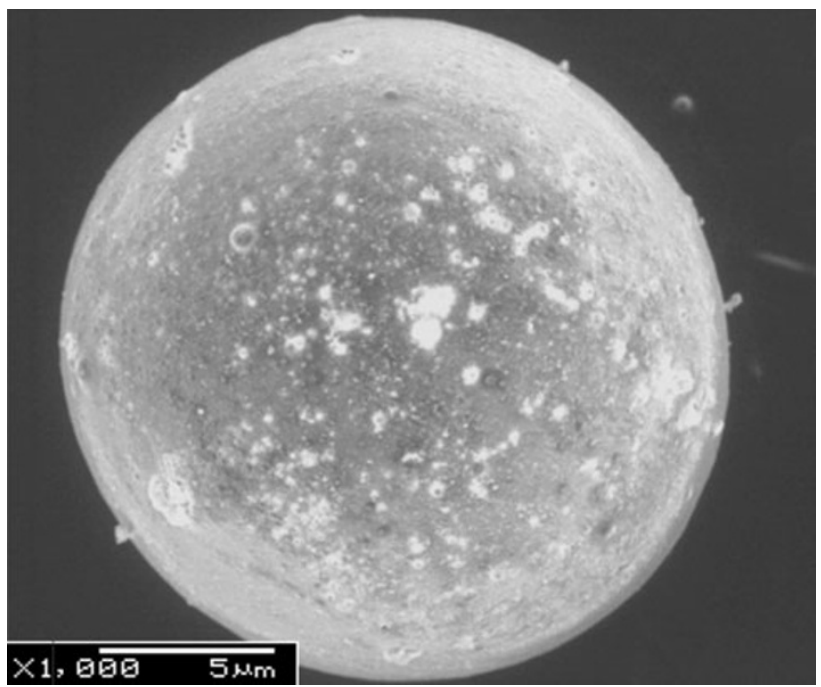

Fig. 5 SEM of MWCNTs-impregnated EC microsphere 
Fig. 6 PXRD pattern of $A$ MT, $B$ MWCNTs, $C$ MWCNTs:MT, $D$ EC microspheres impregnated with MWCNTs and $E$ EC microspheres without MWCNTs

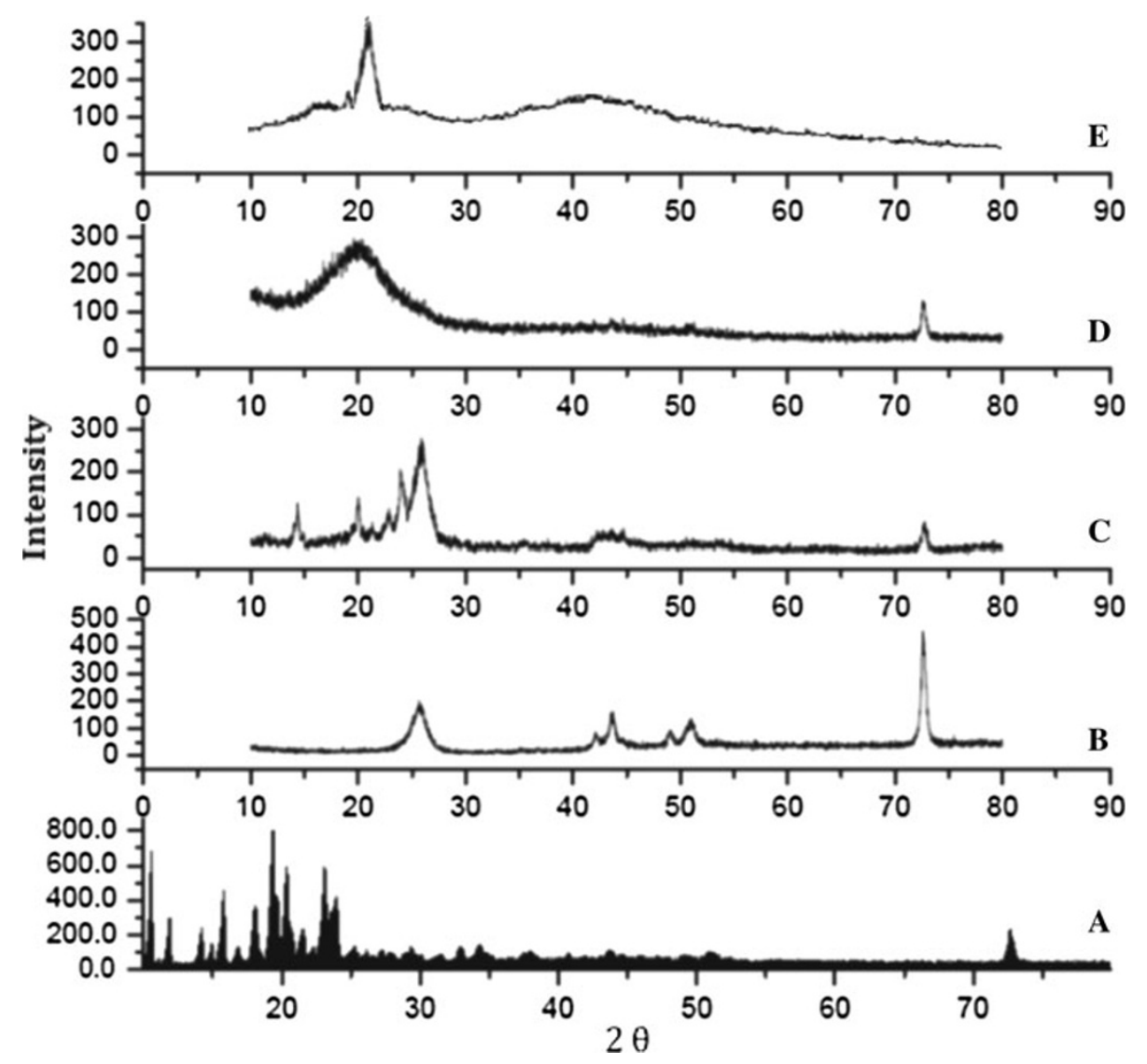

and the possible interaction between drug and other components of microspheres. The DSC curves are shown in Fig. 7. MT showed an endothermic peak at $125.9^{\circ} \mathrm{C}$ corresponding to its melting point and one exothermic peak at $238^{\circ} \mathrm{C}$, which is still under further evaluation. Drug adsorbate also showed a similar characteristic peak with decreased intensity showing its stability during the nanoprecipitation process. The DSC curve of adsorbate had very little shift toward the lower side, which attributes physical interaction MT over the surface of MWCNTs. There was a total disappearance of endothermic peaks of the drug in drug-loaded microspheres, which supports complete entrapment of drug inside matrix of microspheres.

\section{Dissolution studies}

The release of any drug molecule from the reservoir-type formulation predominantly depends upon pore size of the reservoir, molecular weight and hydrophobicity of the drug molecules. Devices with a larger pore size cannot control the release of drugs effectively from the reservoir. Drug release studies of all batches of microspheres were performed to evaluate the potential ability of CNTs in sustaining the release rate of drug from the microspheres. The dissolution studies of MWCNTs-impregnated microspheres revealed sustained drug release up to $24 \mathrm{~h}$, while microspheres without MWCNTs exhibited 90\% drug release within $8 \mathrm{~h}$ (Fig. 8). Batch 8 was considered as an optimized formulation for sustained release microspheres of MT on the basis of its ability to sustain drug release up to $24 \mathrm{~h}$ with almost $15 \%$ drug release in the first hour. Moreover, it also exhibited the highest value for $t_{50}$ (15.05 hours) and EE (97.78\%) among all batches formulated.

This finding was in close agreement with the results obtained by Zhang et al. (2010) for theophylline. As per literature review, hydrophobic interaction and pore size of the nanometer scale of MWCNTs make it difficult for the dissolution medium to penetrate inside the matrix of microspheres. For highly water-soluble drugs (MT), simple entrapment inside the nanocavity was likely to be effective for producing adequate sustained release property. From the dissolution study, it was considered that nanometerscale diameter of MWCNTs and hydrophobic interaction were the prime factors governing the release of MT. The data obtained for in vitro release were fitted into various release kinetic model equations (zero-order, first-order, Hixson-Crowell, Weibull, Kosemeyer-Peppas and Higuchi release models). The in vitro drug release of optimized batch displayed highest regression coefficient for Higuchi's model indicating diffusion to be the predominant mechanism of drug release (Costa and Manuel 2001; Reza et al. 2003; Gohel et al. 2000). 


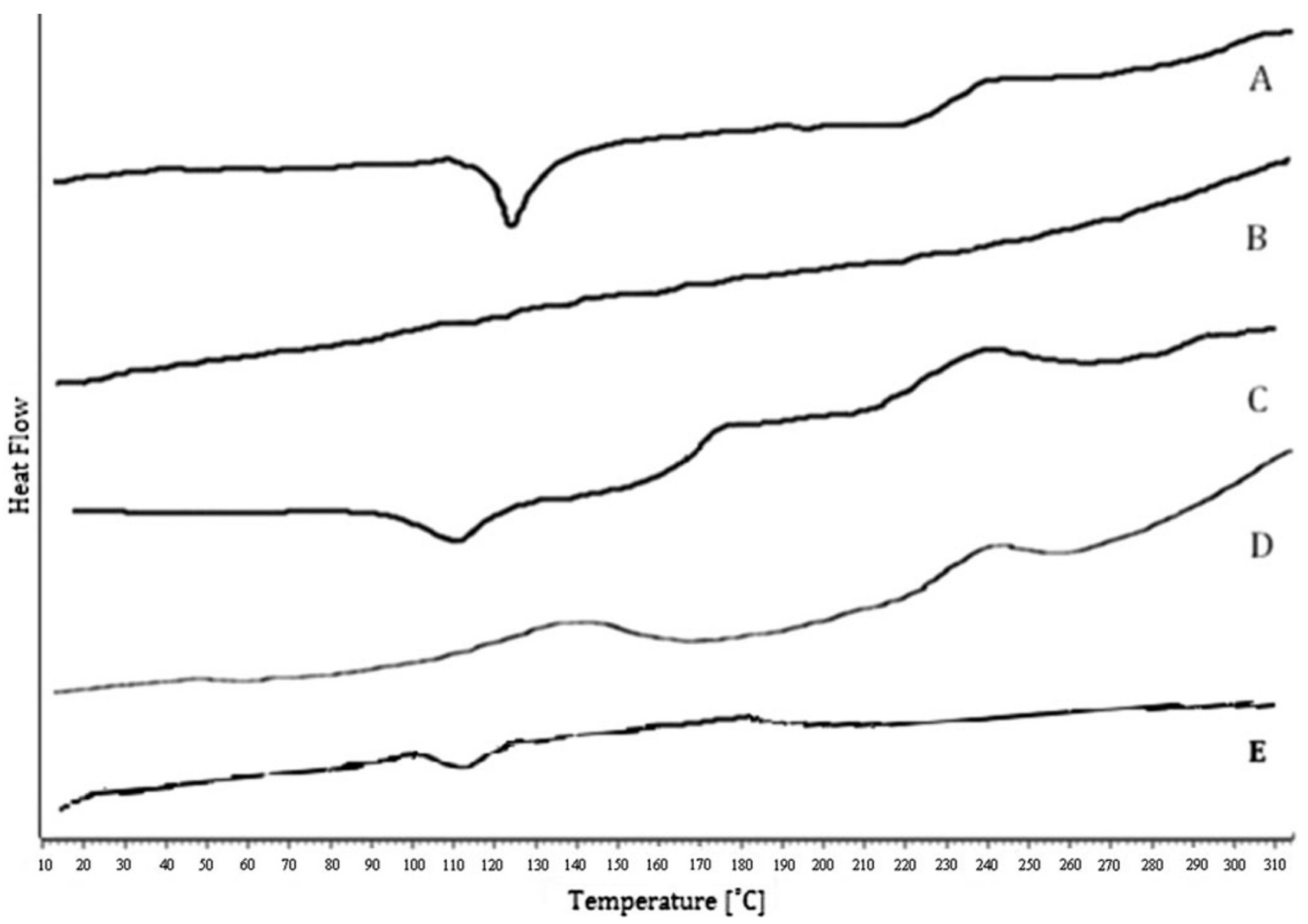

Fig. 7 DSC thermogram of $A$ MT, $B$ MWCNTs, $C$ MWCNTs:MT, $D$ EC microspheres impregnated with MWCNTs and $E$ EC microspheres without MWCNTs

Fig. 8 Comparison of in vitro drug release profiles of EC microspheres with and without impregnation of MWCNTs

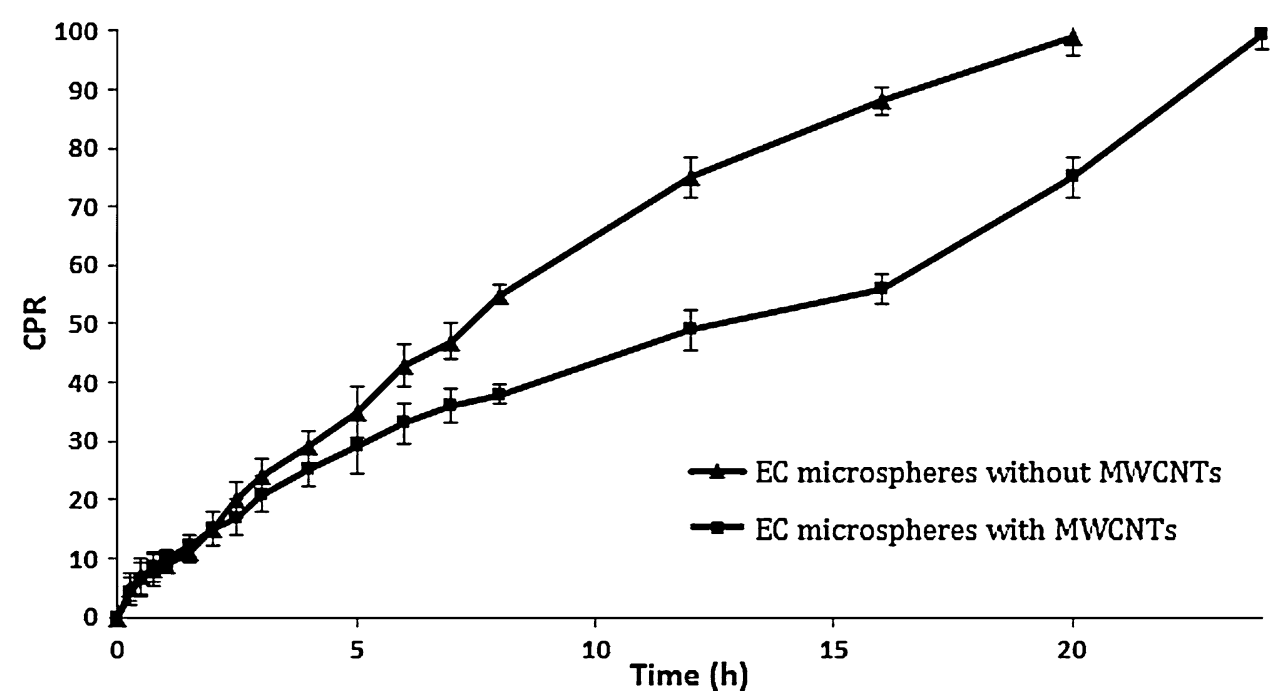

\section{Conclusions}

The present study has been a satisfactory attempt to formulate a sustained release microparticulate system of a highly water-soluble drug, MT, with an objective of minimizing frequency of daily dosing. It can be concluded from experimental results that CNTs-doped EC microspheres revealed high total yield, and the excellent flow 
behavior for all batches was independent of the amount of MWCNTs incorporated. Drug entrapment efficiency of EC microspheres prepared with drug adsorbate was very high for all batches, which favors its use as an excellent carrier for highly water-soluble drugs, like MT. Moreover, the central composite design of the experiment had been successfully employed for optimizing the formulation. The optimized formulation (Batch 8 ) revealed sustained release up to $24 \mathrm{~h}$ with only $15 \%$ drug release at the end of the first hour. it was best fitted to Higuchi model out of all kinetic models applied. Further, the retention characteristics, as well as entrapment efficiency, are far better than all other previous reports. However, the formulation still required an extensive in vivo study before utilizing MWCNTs as a carrier for the products used by human beings.

Acknowledgments The authors would like to thank Sun Pharmaceuticals, Mumbai, India for providing the gift sample of metoprolol tartrate.

Open Access This article is distributed under the terms of the Creative Commons Attribution License which permits any use, distribution and reproduction in any medium, provided the original author(s) and source are credited.

\section{References}

Abdekhodaie MJ, Wu XY (2008) Drug release from ion-exchange microspheres: mathematical modeling and experimental verification. Biomaterials 29:1654-1663

Afolabi A (2006) In-vitro adsorption of fluoroquinolones on some pharmaceutical adsorbents. Tropical J Pharm Res 5:533-538

Aideloje O, Onyeji C, Ugwu N (1998) Altered pharmacokinetics of halofantrine by an antacid, magnesium carbonate Sylvester. Eur J Pharm Biopharm 46:299-303

Ajima K, Yudasaka M, Murakami T, Maigne A, Shiba K, Iijima S (2004) Drug-loaded carbon nanohorns: adsorption and release of dexamethasone in vitro. Mol Pharm 1:399-405

Ajima K, Yudasaka M, Murakami T, Maigne A, Shiba K, Iijima S (2005) Carbon nanohorns as anticancer drug carriers. Mol Pharm 2:475-480

Alkhamis K (2001) Adsorption of allopurinol and ketotifen by chitosan. AAPS PharmSciTech, article 2

Allemann E, Gurny R, Doelker E (1993) Drug-loaded nanoparticles: preparation and drug targeting issues. Eur J Pharm Biopharm 39:173-191

Amperiadou A, Georgarakis M (1995a) Controlled release salbutamol sulphate microcapsules prepared by emulsion solvent-evaporation technique and study on the release affected parameters. Int $\mathbf{J}$ Pharm 115:1-8

Amperiadou A, Georgarakis M (1995b) Preparation and characterization of ethyl cellulose walled theophylline microcapsules using the emulsion solvent evaporation technique. Drug Dev Ind Pharm 21:1339-1346

Anal AK, Stevens WF (2006) Ionotropic cross-linked chitosan microspheres for controlled release of ampicillin. Int J Pharm 312:166-173

Bajpai SK, Saxena S (2004) Dynamic release of riboflavin from a starch-based semi IPN via partial enzymatic degradation: part II. React Funct Polym 59:115-129
Benita S, Donbrow M (1982) Effect of polyisobutylene on ethylcellulose-walled microcapsules: wall structure and thickness of salicylamide and theophylline microcapsules. J Pharm Sci 71:205-210

Benita S, Benoit JP, Puisieux F, Theis C (1984) Characterization of drug-loaded poly(D,L-lactide) microspheres. J Pharm Sci 73:1721-1724

Blandino A, Macias M, Cantero D (2000) Glucose oxidase release from calcium alginate gel capsules. Enzyme Microb Technol 27:319-324

Blandino A, Macias M, Cantero D (2001) Immobilization of glucose oxidase within calcium alginate gel capsules. Process Biochem 36:601-606

Bodmeier R, McGinity JW (1987a) Polylactic acid microspheres containing quinidine base and quinidine sulphate prepared by the solvent evaporation technique. II. Some process parameters influencing the preparation and properties of microspheres. J Microencapsul 4:289-293

Bodmeier R, McGinity JW (1987b) The preparation and evaluation of drug-containing poly(D,L-lactide) microspheres formed by the solvent evaporation method. Pharm Res 4:465-471

Bonard JM, Forro L, Ugarte D, De Herr WA, Chatelain A (1998) Physics and chemistry of carbon nanostructures. Eur Chem Chron 3:9-16

Boonsongrit Y, Abe H, Sato K, Naito M, Yoshimura M, Ichikawa H, Fukumori Y (2008) Controlled release of bovine serum albumin from hydroxyapatite microspheres for protein delivery system. Mat Sci Eng B 148:162-165

Cheu SJ, Chen RRL, Chen PF, Lin WJ (2001) In vitro modified release of acyclovir from ethyl cellulose microspheres. J Microencapsul 18:559-565

Choi JS, Han HK (2005) Enhanced oral exposure of diltiazem by the concomitant use of naringin in rats. Int $\mathbf{J}$ Pharm 305:122128

Choudhury PK, Kar M (2009) Controlled release metformin hydrochloride microspheres of ethyl cellulose prepared by different methods and study on the polymer affected parameters. J Microencapsul 26:46-53

Chowdary KPR, Rao NK, Malathi K (2004) Ethyl cellulose microspheres of glipizide: characterization, in vitro and in vivo evaluation. Indian J Pharm Sci 66:412-416

Chrysant SG (1998) Fixed low-dose drug combination for the treatment of hypertension. Arch Fam Med 7:370-376

Costa P, Manuel J (2001) Modeling and comparison of dissolution profiles. Eur J Pharm Sci 13:123-133

Dadashzadeh S, Zarghi A, Ebrahimian A (2003) Pharmacokinetics and comparative bioavailability of two diltiazem tablet formulations in healthy volunteers. DARU 11:14-18

Davydov VY, Kalashnikova EV, Karnatsevich VL, Kirillov AI (2005) Adsorption properties of multi-wall carbon nanotubes. Fuller Nanotubes Carbon Nanostructures 12:513-518

Galeone M, Nizzola L, Cacioli D, Mosie G (1981) In vitro demonstration of delivery mechanism from sustained-release pellets. Curr Ther Res 29:217-234

Gambhire MN, Ambade KW, Kurmi SD, Kadam VJ, Jadhav KR (2007) Development and in vitro evaluation of an oral floating matrix tablet formulation of diltiazem hydrochloride. AAPS PharmSciTech 8:E1-E9

Genta I, Perugini P, Conti B, Pavanetto F (1997) A multiple emulsion method to entrap a lipophilic compound into chitosan microspheres. Int J Pharm 152:237-246

Ghebre-Sellassie I (1994) Multiple oral drug delivery. Marcel Dekker, New York

Ghorab MM, Zia H, Luzzi LA (1990) Preparation of controlled release anticancer agents I: 5-fluorouracil-ethyl cellulose microspheres. J Microencapsul 7:447-454

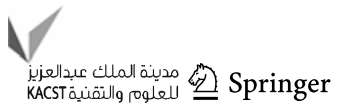


Gohel MC, Panchal MK, Jogani VV (2000) Novel mathematical method for quantitative expression of deviation from the Higuchi model. AAPS PharmSciTech 1:1-6

Hazrati M, DeLuca P (1989) 5-Fluorouracil in biodegradable polymeric microspheres. In: Proceedings of the 16th international symposium on bioactive materials, pp 79-80

Iijima J (1991) Helical microtubules of graphitic carbon. Nature 354:56-58

Ito F, Fujimori H, Makino K (2008) Factors affecting the loading efficiency of water soluble drugs in PLGA microspheres. Colloids Surf B Biointerface 61:25-29

Jadhav N, Pawar A, Paradkar A (2007) Design and evaluation of deformable talc agglomerates prepared by crystallo-co-agglomeration for generating heterogeneous matrix. AAPS PharmSciTech 8:1-7

Jeffery H, Davis SS, O'Hagan DT (1991) The preparation and characterization of poly(lactide-co-glycolide) microparticles 1 . Oil-in-water emulsion solvent evaporation. Int $\mathrm{J}$ Pharm 77:169-175

Jeffery H, Davis SS, O'Hagan DT (1993) The preparation and characterization of poly(lactide-co-glycolide) microparticles. 2. The entrapment of a model protein using a (water-in-oil-inwater emulsion solvent evaporation technique. Pharm Res 10:362-368

Jeyanthi R, Panduranga Rao K (1988) A novel method of preparation of gelatin microspheres for controlled release of anticancer drugs. In: Proceedings of the 15 th international symposium on controlled release of bioactive materials, pp 172-173

Jiang Z, Xu S, Lu Y, Yuan W, Wu H (2006) Nanotube-doped alginate gel as a novel carrier for BSA immobilization. J Biomater Sci Polymer Edn 17:21-35

Lee YSL, Poynter R, Podczeck F (2000) Development of a dual approach to assess powder flow from avalanching behavior. AAPS PharmSciTech 1:1-9

Li YH, Wang S, Wei J, Zhang X, Xu C, Luan Z, Wu D, Wei B (2002) Lead adsorption on carbon nanotubes. Chem Phys Lett 357:263-266

Liu Y, Zhang P, Feng N, Zhang X, Wu S, Zhao J (2009) Optimization and in situ intestinal absorption of self-microemulsifying drug delivery system of oridonin. Int J Pharm 365:136-142

Long RQ, Yang RT (2001) Carbon nanotubes as superior sorbent for dioxin removal. J Am Chem Soc 123:2058-2059

Lu Y, Xu S, Jiang Z, Yuan W, Wang T (2005) Diffusion of nicotinamide adenine dinucleotide in calcium alginate hydrogel beads doped with carbon and silica nanotubes. J Chem Eng Data 50:1319-1323

Luzzi LA, Palmier A (1984) An overview of pharmaceutical applications of microencapsulation. CRC Press, Boca Raton

Martindale SS (2009) The complete drug reference. Pharmaceutical Press, London

Niyogi S, Hamon MA, Hu H, Zhao B, Bhowmik P, Sen R, Itkis ME, Haddon RC (2002) Chemistry of single-walled carbon nanotubes. Acc Chem Res 35:1105-1113

O'Donnell PB, McGinity JW (1997) Preparation of microspheres by the solvent evaporation technique. Adv Drug Dev Rev 28:25-42
Parul T, Garud N, Verma A (2008) Preparation and characterization of aceclofenac microspheres. Asian J Pharm 2:110-115

Patel A, Subhabrata R, Thakur RS (2006) In vitro evaluation and optimization of controlled release floating drug delivery system of metformin hydrochloride. DARU 14:57-64

Peng X, Li Y, Luan Z, Di Z, Wang H, Tian B, Jia Z (2003) Adsorption of 1,2-dichlorobenzene from water to carbon nanotubes. Chem Phys Lett 376:154-158

Reza MS, Quadir MA, Haider SS (2003) Comparative evaluation of plastic, hydrophobic and hydrophilic polymers as matrices for controlled-release drug delivery. J Pharm Pharmaceut Sci 6:282-291

Sah H (1997) Microencapsulation technique using ethyl acetate as a dispersed solvent: effects of its extraction rate on the characteristics of PLAG microspheres. J Control Release 47:233-245

Schlicher EJAM, Postma NS, Zuidema J, Talsma H, Hennink WE (1997) Preparation and characterization of poly(D,L-lactideco-glycolic acid) microspheres containing desferrioxamine. Int J Pharm 153:235-245

Shabaraya AR, Narayanacharyulu R (2003) Design and evaluation of chitosan microspheres of metoprolol tartrate for sustained release. Indian J Pharm Sci 65:250-252

Shinde AJ, Garala KC, More HN (2009) Formulation and evaluation of transdermal patches of metoprolol tartrate. Indian Pharm 84:69-74

Singh Y, Singh P, Rani G (2007) Development and evaluation of floating microspheres of verapamil hydrochloride. Braz J Pharm Sci 43:529-534

Tagmatarchis N, Prato M (2005) Carbon-based materials: from fullerene nanostructures to functionalized carbon nanotubes. Pure Appl Chem 77:1675-1685

Tasis D, Tagmatarchis N, Bianco A, Prato M (2006) Chemistry of carbon nanotubes. Chem Rev 106:1105-1136

Wade A, Weller PJ (1994) Handbook of pharmaceutical excipients. The Pharmaceutical Press, Washington, DC

Wu CS, Liao HT (2007) Study on the preparation and characterization of biodegradable polylactide/multi-walled carbon nanotubes nanocomposites. Polymer 48:4449-4458

Yang CY, Tsai SY, Tsiang RCC (2000) An enhanced process for encapsulating aspirin in ethylcellulose microcapsules by solvent evaporation in an o/w emulsion. J Microencapsul 17:269-277

Yang CY, Tsai SY, Tsiang RC (2001) Encapsulating aspirin into a surfactant-free ethyl cellulose microsphere using non-toxic solvents by emulsion solvent evaporation technique. J Microencapsul 18:223-236

Yang JF, Qiu LY, Jina Y, Zhang JX (2005) Thymosin-loaded enteric microspheres for oral administration: preparation and in vitro release studies. Int J Pharm 30:41-47

Zhang Y, Cheng G, Qu X, Wang Y, Ding Z, Cheng L, Yu QP (2008) Preparation and characterization of galactosylated chitosan coated BSA microspheres containing 5-fluorouracil. Carbohydr Polym 72:390-397

Zhang X, Hui Z, Wan D, Huang H, Huang J, Yuan H, Yu J (2010) Alginate microsphere filled with carbon nanotube as drug carrier. Int J Bio Macromol 47:389-395 